\title{
Understanding COVID-19 from innate immune perspective and develop possible therapeutic interventions.
}

\author{
Rahul Gupta, PhD, \\ rbiochem@gmail.com
}

\section{Summary:}

The present hypothesis is envisioned in trying to develop therapeutic intervention measures against SARS-CoV2, causative agent for COVID-19. Studying the innate immune response to SARS-CoV2 gives us a proper understanding of the pathogenesis phenomena by the virus. Possibly, SARS-CoV2 is suppressing the host interferon system and over stimulating the proinflammatory cytokines, leading to cytokine storms culminating in tissue damages and other ensuing pathophysiologies. Hence, combinatorial approaches using repurposed antiinflammatory and antiviral drugs has been thought of as good intervening measures against COVID-19.Anti- inflammatory for blocking the IL1 $\beta$ production and antivirals ( IFNs or viral polymerase inhibition) for curbing viral load would be perhaps a good combination.

\section{Introduction:}

On Dec 2019, a new infectious respiratory disease (COVID 19) emerged in Wuhan province, China. The etiological agent was identified to be a novel coronavirus, SARS-CoV2, which is closely related to previously known SARS-CoV. Despite drastic containment measures the virus wreaked havoc causing a pandemic, infecting about 27,00,000 people around the world with more than 190,000 fatalities. Coronaviruses (CoV) are positive single stranded enveloped RNA viruses. As the virus is single stranded RNA virus, it's mutation frequency is expected to be much higher than the DNA viruses. SARS-CoV2 are known to bind to ACE2 receptors of target epithelial cells of lung, intestine, blood vessels and initiate infection ${ }^{1}$. As of now there are no specific drug known to effectively manage the virus, hence there is an unmet need on pressing exigency.

\section{Innate immune response to SARS-CoV2:}

The fact that a fraction of the population infected with SARS-CoV2 are asymptomatic, with mild symptoms, and others severely symptomatic raised the question of involvment of innate immune system in understanding the pathogenesis phenomena properly. More so, a proper dissection in this direction would perhaps lead to design strategies for possible intervention against COVID-19.

Following pathogen (virus/bacteria/fungi) sensing by the host pattern-recognition receptors (PRRs), the host induces the production of molecules limiting the growth of pathogens and pro-inflammatory cytokines. The PRRs include Toll like receptors, RIG-I like receptors and 
NOD like receptors. The pro-inflammatory cytokines, when produced in controlled fashion are also capable of protective action against pathogens by limiting their growth. However, the deregulated production of the pro-inflammatory cytokines contributes to inflammation, tissue damages and further ensuing pathophysiologies ${ }^{2}$. It's the fine tuning between the molecules restricting the growth of pathogens and pro-inflammatory molecules which maintains the balance and helps in running the host system effectively.

The interferons (IFNs) provides the first line of innate defence against viral infection in vertebrates. Upon viral infection, host PRRs sense viral components (RNA) and initiate a signaling cascade resulting in the secretion of IFNs. These IFNs further leads to the transcriptional induction of many IFN-stimulated genes (ISGs), out of which many have antiviral activity specifically against RNA viruses ( OAS1, MX1, ISG1, etc). Type 1 IFNs (IFN- $\alpha$, IFN- $\beta$, IFN- $\omega$, IFN- $\varepsilon$, IFN- $\zeta$, IFN- $\kappa$, IFN- $\tau$, and IFN- $\delta$ ) are the most recognised viral inducible cytokine having antiviral properties ${ }^{3}$.Coronaviruses are known to inhibit host Type 1 IFN production, which may account for viral pathogenesis ${ }^{4}$. The other RNA virusesInfluenza virus ${ }^{5}$ Ebola virus ${ }^{6}$ Dengue virus $^{7}$ are also known for suppressing host IFN production for self propagation. Bats are known to constitutively express high amount of IFN- $\alpha$ which may explain the antiviral state behind the ability of bats to host many of these pathogenic viruses asymptomatically ${ }^{8}$. Of the PRRs, TLR7 (endosomes) and RIG-I (cytosolic) like receptor are recognised to be single stranded RNA sensor for IFN production ${ }^{9}$.

Amongst the pro-inflammatory cytokines, (IL1 $\beta$ ) is one of the most known to be induced by RNA viruses thru the involvement of NLRP3 inflammasomes ${ }^{10}$. SARS Coronavirus is also known to activate NLRP3 inflammsomes ${ }^{11}$. The transcriptional priming of NLRP3 transcript was also found to be impaired in bats leading to a defective (IL1 $\beta$ ) production ${ }^{12}$, which might attribute to reason for harbouring these viruses asymptomatically. Clinically, ACE2 (+) lung cells from patients infected with the virus have also shown high expression of proinflammatory cytokines (IL1 $\beta, \operatorname{IL6}, \mathrm{TNF} \alpha)^{13}$.Interestingly, the patients who got infected in China, Italy and Spain had reported comorbidities with diabetes, heart diseases and hypertension. Notably, these pathological conditions are attributed to hyper activated IL1 $\beta$ production $^{14,15,16}$

\section{Possible intervention measures taming the hyper inflammatory cytokine storm:}

Acute COVID-19 patients have shown deposition of fibrin and platelet microthrombi in the lung vasculature, which contribute to progressive respiratory dysfunction and eventual deaths. Abnormal bleedings were also observed in Catheter and IV channels, with increased ferritin level and CRP in blood. These were perhaps due to activation of the coagulation cascades (similar to sepsis), the aftermath of cytokine storm produced by the deregulated immune system. Anti-clotting Tissue Plasminogen activator (tPA) has been shown to very effective in this aspect ${ }^{17}$. Clinical trials to be started soon on this. Coincidently, the sepsis drug (Xigris) was also an anti-coagulant. 
Chloroquine/Azithromycin combination has seen some mixed efficacy data against SARSCoV2. Azithromycin, a macrolide antibiotic is much known to be a bacterial translational inhibitor, specifically for gram negatives. Azithromycin is also known to exert antiviral activity in cystic fibrosis patients by the induction of IFNs and ISGs ${ }^{18}$. Perhaps in COVID-19 patients too, the antibiotic takes care of secondary bacterial infections which develop with the host system becoming compromised following the cytokine storm.

IL6 Receptor blocker Tocilizumab is also in contention as intervention measure against SARS-CoV2. Interestingly, IL6 KO mice showed increased mortality with Influenza virus and HSV-1 virus challenge, conferring a protective action of IL6 ${ }^{19,20}$. Bacterial endotoxin induced ARDS also showed reduced pulmonary inflammation with recombinant IL6, reconfirming the protective action of the cytokine ${ }^{21}$. However, perhaps it's the timing and the amount of induction of the cytokine which decides whether it is going to be protective or detrimental to the system. It's really tempting to speculate how IL6 blocker turns up as intervention measure in clinical trials.

TLR7 is a single stranded RNA sensor located in the endosomes of lung epithelial cells and airway smooth muscles. TLR7 has been of importance in asthma, the disorder of lungs characterized by hyperactivated Th2 adaptive response (excessive broncho constriction and inflammation). The TLR7 agonist Imiqimod (R837) has found therapeutic application in attenuating airway inflammation by reducing the Th2 response ( IL4, 1L5, 1L13) and shifting the equilibrium towards activating Th1 response ( IFNS, ISGs) ${ }^{22}$. Imiqimod might be helpful in reversing the airway hyper responsiveness of COVID-19 patients too.

IL1 $\beta$, a pro-inflammatory cytokine is known to contribute towards inflammation (sterile and pathogen mediated). Hyper stimulation of the cytokine leads to adverse pyroptosis and cell death. Sterile inflammation leads to different pathological disorders like Rhematoid arthiritis, Gout, Diabetes, Alzheimers, Atherosclerosis, etc. Blocking IL1 $\beta$ production by IL1R antagonist Anakinra have shown promising results in these conditions ${ }^{23,24}$. In COVID-19 patients too, the IL1 $\beta$ production is robust leading to cytokine storm, which attribute to hyper inflamed state and pathophysiologies. Hence, IL1 $\beta$ inhibitor might be helpful in the management of COVID-19.Combinatorial approaches using anti-inflammatories and antiviral repurposed drug (anti-inflammatories for dampening the IL1 $\beta$ production and antivirals for curbing the viral load with IFNs or viral polymerase inhibition) might be good intervening measures.

Type 1 IFN has been shown to play protective role in SARS-CoV2 pathogenesis in human bronchial epithelial cells and A549 expressing ACE2. COVID-19 patients were seen to express high levels of C-X-C Chemokines (CXCL2, CXCL8, CXCL9, CXCL16) and Interleukins (IL1 $\beta$ and IL6) ${ }^{25}$. The Chemokines are also being known to be positively regulated by IL1 $\beta$, along with neutrophil infiltration in lungs during inflammations ${ }^{26}$. Autopsies of COVID-19 lungs have shown to be filled with jelly like material causing severe respiratory distress probably by the accumulation of hyaluronic acid. And, the proinflammatory cytokines IL1 $\beta$ and TNF $\alpha$ are known to be inducers for hyaluronic acid ${ }^{27}$. 
Summing up together the following combinatorial approaches may be tried empirically :

1) Recombinant Type I interferon as antiviral, in combination with a IL1 $\beta$ inhibitor as anti-inflammatory (Anakinra)

2) TLR7 agonist Imiqimod as antiviral, in combination with a IL1 $\beta$ inhibitor as antiinflammatory (Anakinra).

3) IL1 $\beta$ inhibitor (Anakinra) as anti-inflammatory, in combination with CoV RNA Polymerase inhibitor (Remdesivir/ Favipiravir)

The patients who are mildly asymptomatic, in whom the the cytokine storm hasn't started or not caused enough damage to be managed perhaps would be the best respondent of these inteventions. While, asymptomatic patients should only need antivirals.

\section{Conclusion:}

The present hypothesis is postulated on the fact that a fraction of the population which get infected by SARS-CoV2 remain asymptomatic or mildly infected, while another fraction being severely symptomatic. In the asymptomatic or mildly infected population, the proinflammatory cytokines perhaps play a protective role. On the contrary, in the severely infected population, the pro-inflammatory cytokines are detrimental, causing the cytokine storm with increasing viral load, tissue damages and pathophysiologies. Coincidently, the patients in Europe who fell sick have reported comorbidities (diabetes, heart disease, hypertension) with high IL1 $\beta$ production. The intervention measures which have been hypothesised are combinatorial approaches by blocking the hyper activated IL1 $\beta$ production along with stimulation of the type 1 IFNs or viral polymerase inhibitor. These interventions can be perhaps tried for other deadly zoonotic RNA viruses, which evade the host immune surveillance for self propagation. However, further basic science research is required to explore these intervention measures and large scale clinical trials to validate it along.

\section{References:}

1 Fang L, Karakiulakis G, Roth M. Are patients with hypertension and diabetes mellitus at increased risk for COVID-19 infection? Lancet Respiratory Medicine 2020; 8: e21

2 Jensen S and Thomsen AR. Sensing of RNA Viruses: a Review of Innate Immune Receptors Involved in Recognizing RNA Virus Invasion. J Virol 2012; 86: 2900-2910

3 Banerjee A, Baker ML, Kulcsar K, Misra V, Plowright R, Mossman K. Novel Insights Into Immune Systems of Bats. Front Immunol 2020; 11: 1-15

$4 \mathrm{Hu}$ Y, Li W, Gao T et al. The Severe Acute Respiratory Syndrome Coronavirus Nucleocapsid Inhibits Type I Interferon Production by Interfering with TRIM25Mediated RIG-I Ubiquitination. J Virol 2017; 91: e02143-16.

5 Jia D, Rahbar R, Chan RWY et al. Influenza Virus Non-Structural Protein 1 (NS1) Disrupts Interferon Signaling. Plos One 2010; doi.org/10.1371/journal.pone.0013927.

6 Cardenas WB, Loo YM, Gale M et al. Ebola Virus VP35 Protein Binds DoubleStranded RNA and Inhibits Alpha/Beta Interferon Production Induced by RIG-I Signaling. J Virol 2006; 80: 5168-5178. 
7 Munos-Jordan JL, Sanchez-Burgos GG, Laurent-Rolle M, Garcia-Sastra A. Inhibition of interferon signaling by dengue virus. PNAS 2003; 100: 14333-338.

8 Zhou P, Tachedjian M, Wynne JW et al. Contraction of the type I IFN locus and unusual constitutive expression of IFN- $\alpha$ in bats. PNAS 2016; 113: 2696-2701.

9 Crozat $\mathrm{K}$ and Beutler B. TLR7: A new sensor of viral infection. PNAS 2004; 101: 6835-36.

10 da Costa LS, Outlioua A, Anginot A, Akarid K, Arnoult D. RNA viruses promote activation of the NLRP3 inflammasome through cytopathogenic effect-induced potassium efflux. Cell death and disease 2019; 10:1-15.

11 Chen IY, Moriyama M, Chang MF, Ichinohe T. Severe Acute Respiratory Syndrome Coronavirus Viroporin 3a Activates the NLRP3 Inflammasome. Front Microbiol 2019; doi: 10.3389/fmicb.2019.00050

12 Ahn M, Anderson DE, Zhang Q, Tan CW et al. Dampened NLRP3-mediated inflammation in bats and implications for a special viral reservoir host. Nature Microbiology 2019; 4: 789-99.

13 He L, Ding Y, Zhang Q, Che X et al .Expression of elevated levels of proinflammatory cytokines in SARS-CoV-infected ACE2+ cells in SARS patients: relation to the acute lung injury and pathogenesis of SARS. J Pathol 2006; 210: 28897.

14 Boni-Schnetzler M, Thorne J, Parnaud G, Marselli L et al. Increased Interleukin (IL)$1 \beta$ Messenger Ribonucleic Acid Expression in $\beta$-Cells of Individuals with Type 2 Diabetes and Regulation of IL-1 $\beta$ in Human Islets by Glucose and Autostimulation. $J$ Clin Endocrinol Metab 2008 ; 93: 4065-4074.

15 Krishnan SM, Sobey CG, Latz E, Mansell A, Drummond GR. IL-1 $\beta$ and IL-18: inflammatory markers or mediators of hypertension? Br J Pharmacol 2014; 171: 5589-5602.

16 Duewell P, Kono H, Rayner KJ, Sirois CM et al. NLRP3 inflamasomes are required for atherogenesis and activated by cholesterol crystals that form early in disease. Nature 2010; 464: 1357-1361.

17 Wang J, Hajizadeh N, Moore EE, Mcylntyre RC et al. Tissue Plasminogen Activator (tPA) Treatment for COVID-19 Associated Acute Respiratory Distress Syndrome (ARDS): A Case Series. Journal of thrombosis and haemostasis 2020. https://doi.org/10.1111/jth.14828.

18 Schogler A, Kopf BS, Edwards MR, Johnston SL et al. Novel antiviral properties of azithromycin in cystic fibrosis airway epithelial cells. European Respiratory Journal 2015 ; 45: 428-439.

19 Dienz O, Rud JG, Eaton SM, Lanthier PA et al. Essential role of IL-6 in protection against H1N1 influenza virus by promoting neutrophil survival in the lung. Mucosal immunology 2012; 5: 258-66.

20 Murphy EA, Mark Davis J, Brown AS, Carmicheal MD, Ghaffar A and Mayer EP. Effect of IL-6 Deficiency on Susceptibility to HSV-1 Respiratory Infection and Intrinsic Macrophage Antiviral Resistance. Journal of Interferon and Cytokine Research 2008 https://doi.org/10.1089/jir.2007.0103 
21 Voiriot G,Razazi K, Amsellem V, Nhieu JTV et al. Interleukin-6 displays lung antiinflammatory properties and exerts protective hemodynamic effects in a double-hit murine acute lung injury. Respiratory Research 2017. DOI 10.1186/s12931-0170553-6.

22 Drake MG, Kaufman EH, Fryer AD,Jacoby DB. The Therapeutic Potential of Tolllike Receptor 7 Stimulation in Asthma. Inflamm Allergy Drug Targets 2012; 11: 484491.

23 Dinarello CA, Simon A, Van der Meer JWM. Treating inflammation by blocking interleukin-1 in a broad spectrum of diseases. Nat Rev Drug Discov 2012; 11: 633652.

24 Larsen CM, Faulenbach M, Vaag A, Vølund A et al. Interleukin-1-receptor antagonist in type 2 diabetes mellitus. N Engl J Med 2007; 356:1517-26.

25 Bianco Mello D, Nilsson Payant BE, Liu WC, Uhi S et al. Imbalanced host response to SARS-CoV-2 drives development of COVID-19. Cell 2020; DOI: 10.1016/j.cell.2020.04.026

26 Calkins CM, Bensard DD, Shames BD, Pulido EJ et al. IL-1 regulates in vivo C-X-C chemokine induction and neutrophil sequestration following endotoxemia. Journal of Endotoxin Research 2002; 8: 59-67.

27 Shi Y, Wang Y, Shao C, Huang J et al. COVID-19 infection: the perspectives on immune responses. Cell Death and Differentiation 2020; 27: 1451-1454

\section{Contributions:}

RG did the literature survey, hypothesised the concept and wrote the manuscript.

\section{Acknowledgment:}

RG is sincerely grateful to Dr Kate Fitzgerald and Dr Douglas Golenbock ( UMASSMED) for the insightful discussions on the concept.

\section{Funding Source:}

The Study hasn’t received any funding as yet.

\section{Declaration of Interest:}

I don't have any competing interest. 
